\title{
Metastasis of Solid Tumors in Bone Marrow: A Study from Northern India
}

\author{
Syed Riaz Mehdi • Madan Lal Brahma Bhatt
}

Received: 23 October 2010/Accepted: 12 April 2011/Published online: 17 May 2011

(C) Indian Society of Haematology \& Transfusion Medicine 2011

\begin{abstract}
The metastasis of bone marrow by the solid tumors is a sign of advanced stage of disease and poor prognosis. The aim of this study was to assess the pattern of bone marrow involvement of different solid tumors and their correlation with hematological findings. In a retrospective study we evaluated 434 aspirates and 76 biopsy sections from 124 cases of different types of solid tumors previously diagnosed on the basis of their clinical and histopathological findings. The hematological profile of the patients was done and correlated with the bone marrow findings. The study was carried out at a medical college hospital of northern India. Out of 124 cases of solid tumors $31(25 \%)$ have metastasized to bone marrow. The highest number $25(36 \%)$ of bone marrow involvement was seen in carcinoma prostate followed by gastric carcinoma and melanoma (25\%) The least number (14.2\%) cases of bone marrow metastasis were observed in endometrial
\end{abstract}

The work was carried out at Department of Pathology and Radiotherapy, JN Medical College, AMU, Aligarh, UP, India.

S. R. Mehdi $(\square)$

Department of Pathology JNMC, AMU, Aligarh, India

e-mail: riaz_mehdi@hotmail.com

Present Address:

S. R. Mehdi

Department of Medical Laboratory, Medical College of Health (Men), King Saud University, Riyadh, India

M. L. B. Bhatt

Department of Radiotherapy, JNMC, AMU, Aligarh, India

e-mail:drmlbbhatt@yahoo.com

Present Address:

M. L. B. Bhatt

Department of Radiotherapy, C.S.M Medical University,

Lucknow, India carcinoma. Anemia was found the commonest (71.4\%) hematological finding followed by thrombocytopenia in $45.1 \%$ cases. The bone marrow examination is an easy, convenient, sensitive and cost effective procedure for assessment of staging and monitoring of prognosis of solid tumors.

Keywords Solid tumors - Bone marrow - Metastasis . Prognosis

\section{Introduction}

The bone marrow in addition to being the origin of primary hematological malignancies is also commonly involved by metastatic solid tumors.

In the last six decades many studies have been carried out in different parts of the world on bone marrow invasion by solid tumors [1-3].

Bone marrow examination; aspiration and biopsy have been well known procedures to diagnose hematological malignancies. Beside this the bone marrow examination is a commonly employed tool for staging and prognosis of various solid tumors $[4,5]$.

Some of the authors have reported that demonstration of tumor cells in marrow might be the first available evidence of malignant solid tumors [4].

The malignancies of prostate, breast, lungs, kidney, thyroid and gastric in adults and neuroblastoma and rhabdomyosarcoma in children are the primary tumors which frequently involve bone marrow [6-12].

The bilateral bone marrow biopsy is supposed to be more accurate procedure for reporting of metastasis of solid tumors. The metastasis is also frequently reported on examination of aspirates only [3]. 
The presence of metastasis in the bone marrow is usually incurable but not necessarily fatal. It is therefore considered imperative to rule out marrow involvement in any malignancy where curative treatment is considered [13].

Bone marrow aspiration is an easy and cost effective method of reporting of metastasis in a very short span of time. The patients of solid tumors with bone marrow metastasis usually present with anemia, leucopenia or pancytopenia [7].

\section{Materials and Methods}

In a retrospective study we evaluated 434 bone marrow aspirates and biopsies from 124 patients of solid tumors previously diagnosed on the basis of clinical and histopathological examinations. The study was carried out in JN medical college, Aligarh, India.

The bone marrow aspirates and biopsies were collected from posterior iliac crest using a Jamshidi aspiration and trephine needles. 8-10 smears were prepared by push and squash techniques. Five methanol fixed smears were stained by Leishman's stain and the remaining unstained smears were preserved for special stains, if and when required. A core of $1.5 \mathrm{~cm}$ was drawn and processed in histopathology lab. 3-4 $\mu \mathrm{m}$ thick sections were stained by Hematoxylene and Eosin $(\mathrm{H} \&$ E). Trephine biopsy specimens were available for only 76 out of 124 patients.

$3 \mathrm{ml}$ blood samples were collected in EDTA. The blood was analyzed on a hematology analyzer. Blood was tested for hemoglobin, total RBC count (TRBC), total leukocyte count (TLC), Platelet count, and red cell indices; mean cell volume (MCV), mean cell hemoglobin $(\mathrm{MCH})$ and mean cell hemoglobin concentration (MCHC). Wright's stained peripheral blood smears were examined under microscope for blood cell morphology.

\section{Results}

In our study of 124 patients $80(64.5 \%)$ were males and 44 $(35.5 \%)$ were females. We evaluated 434 aspirates from 124 patients. Based on clinical and histopathological diagnosis, 36 (29.0.\%) were carcinoma breast, $25(23.3 \%)$ carcinoma prostate, $19(15.3 \%)$ carcinoma lung, 20 (16.1\%) gastric carcinoma, $5(4.0 \%)$ Ewing's sarcoma, 4 (3.2\%) melanoma, $4(3.2 \%)$ carcinoma of thyroid, 7 (5.6\%) endometrial carcinoma and $4(3.2 \%)$ with unknown primary site.

Bone marrow metastasis was found in 31 out of 124 (25\%) malignant solid tumors.
Table 1 Hematological profile of solid tumors with bone marrow metastasis

\begin{tabular}{lcl}
\hline Blood picture & Number of cases & Percentage \\
\hline Anaemia & 22 & 71.4 \\
Thrombocytopenia & 14 & 45.1 \\
Pancytopenia & 6 & 19.3 \\
Bleeding manifestations & 5 & 16.1 \\
\hline
\end{tabular}

Table 1 shows the hematological profile of the cases of solid tumors with metastasis to bone marrow. With 22 out of $31(71.4 \%)$, anaemia was the commonest clinical presentation in patients of solid tumors with bone marrow metastasis. The other hematological manifestations were, thrombocytopenia in $14(45.1 \%)$, pancytopenia in 6 $(19.3 \%)$ and bleeding tendency in $5(16.1 \%)$ cases.

Table 2 shows the number and percentage of cases of each type of solid tumor which has metastasized to bone marrow. 9 out of 25 (36\%) cases of carcinoma Prostate had evidence of metastasis to bone marrow, making it the solid tumor with highest percentage of bone marrow metastasis. Gastric carcinoma and melanoma shared second position with $25 \%$ of tumors having metastasis to bone marrow. The lowest percentage, 1 out of $7(14.2 \%)$ of bone marrow metastasis was observed in endometrial carcinoma. The rest of the tumors came in between as shown in Table 2 .

\section{Discussion}

Bone marrow is an important and frequently involved site of metastasis for solid tumors [6-12]. The advanced diagnostic procedures like bone scan and MRI are more

Table 2 Number and percentage of solid tumors with secondary metastasis to bone marrow

\begin{tabular}{|c|c|c|c|}
\hline $\begin{array}{l}\text { Type of } \\
\text { solid tumor }\end{array}$ & $\begin{array}{l}\text { Number of cases } \\
\text { of primary } \\
\text { malignancy } \\
n=124\end{array}$ & $\begin{array}{l}\text { Number of cases } \\
\text { of metastasis to } \\
\text { B.M } n=31\end{array}$ & $\begin{array}{l}\text { Percentage of } \\
\text { cases of B.M } \\
\text { metastasis } \\
25 \%\end{array}$ \\
\hline Ca. breast & 36 & 8 & 22.2 \\
\hline Ca. prostate & 25 & 9 & 36 \\
\hline SCC lung & 19 & 4 & 21 \\
\hline $\begin{array}{l}\text { Gastric } \\
\text { carcinomas }\end{array}$ & 20 & 5 & 25 \\
\hline Ewing's tumor & 5 & 1 & 20 \\
\hline Melanoma & 4 & 1 & 25 \\
\hline $\begin{array}{l}\text { Endometrial } \\
\text { Ca. }\end{array}$ & 7 & 1 & 14.2 \\
\hline Ca. U. bladder & 4 & 1 & 20 \\
\hline $\begin{array}{r}\text { Unknown } \\
\text { primary }\end{array}$ & 4 & 1 & 20 \\
\hline
\end{tabular}

B.M Bone Marrow, Ca. Carcinoma, SCC S Squamous cell carcinoma 
sensitive to assess the extent of bone marrow metastasis but the procedures are available only at advanced medical centers in cities and are expensive too. While the bone marrow examination is a simple and cost effective procedure and remains the best option to look for bone marrow metastasis of solid tumors and to monitor the prognosis [710]. Bone marrow examination has another advantage in those cases where patients with symptoms of malignancy or with pathological fractures present without clinically detectable primary site of origin. In these cases morphological study of Disseminated Tumor Cell (DTC) helps in assessment of the primary site of solid tumor [13].

The hematogenous spread of tumor cells makes it easier for secondaries to reach the bone marrow. The bone marrow metastasis affects the normal haemopoiesis leading to myelopthesic anaemia and other cytopenias [10]. Advanced stages of bone marrow infiltration by the tumor cells result in thrombocytopenia and bleeding manifestations. We too have come across similar clinical presentation and hematological profile in cases with bone marrow metastasis. Majority of the solid tumors with no involvement of bone marrow were devoid of cytopenias and bleeding manifestations.

The identification of metastatic cells in bone marrow do not pose much of a problem because the cells appear alien to the normal hemopoietic tissue, however sometimes their morphology may not reveal exact site of origin. The morphology of metastatic tumor cells should be correlated with the clinical presentation and histopathological findings of the tumor to clinch the final diagnosis. The presence of tumor cells in the bone marrow is less of a diagnostic and more of a prognostic feature.

In accordance with previous studies from India $[9,10]$ and other parts of the world $[7,8,11-13]$ we too found that carcinoma prostate $(36 \%)$ is the solid tumor which most commonly metastasize to bone marrow, followed by gastric carcinoma and melanoma (25\%). The carcinoma breast metastasis in the present study was not as high as reported earlier from India [9]. The most infrequent involvement of bone marrow (14.2\%) was observed in endometrial carcinoma. Significantly the overall percentage of bone marrow metastasis of only $25 \%$ in our study was much lower than reported from Japan [8] and USA [6-11]. The possible explanation may be that the bone marrow examination was performed there at an advanced stage of the disease while we carried out the procedure little earlier before the infiltration of the marrow took place. Beside this there are other factors governing the course of the solid tumors and their metastasis to bone marrow.

The examination of bone marrow smear and biopsy has always been a good modality for staging and monitoring the prognosis and treatment in cases of malignant solid tumors [13]. The correlation of hematological findings especially the degree of thrombocytopenia with the extent of bone marrow metastasis provides a good overview about the survival of patients $[4,7]$.

\section{References}

1. Reich C (1935) A study of the diagnostic value of sternal puncture in clinical hematology. Am J Med 189:515-520

2. Motulsky AG, Rohn RJ (1953) The bone marrow in metastatic melanoma. J Lab Clin Med 41:526-533

3. Valdes SM, Nava OA, Palacios RV et al (2000) Diagnoses of bone marrow metastasis in children with solid tumors and lymphoma; Aspiration or unilateral or bilateral biopsy? Arch Med Res 3(1):58-61

4. Kanhere MH, Sharma YR, Kalra A (1988) Role of bone marrow examination in staging of carcinoma breast. Indian J Surg 1:54-58

5. Landys K (1982) Prognostic value of bone marrow biopsy in breast cancer. Cancer 49:513-518

6. Anner RM, Drewinko B (1977) Frequency and significance of bone marrow involvement by metastatic solid tumors. Cancer 39:1337-1344. doi:10.1002/1097-0142(197703)39:3<1337:AIDCNCR2820390349>3.0.CO;2-X

7. Kilickap S, Erman M, Dincer M, Aksoy S, Hakan H, Yalcin Y (2007) Bone marrow metastasis of solid tumors: clinicopathological evaluation of 73 cases. Turk J Cancer 37(3):85-88

8. Moriwaki SMK, Okabe K, Yamauchi M, Yamamoto T, Kamei T (2003) Pathological analysis for bone marrow metastasis by aspiration and core needle biopsy. Jpn J Cancer Clinic 49(3): 203-209

9. Mohanty SK, Dash S (2003) Bone marrow metastasis in solid tumors. Indian J Pathol Microbiol 46(4):613-616

10. Tasleem RA, Chowdhary ND, Kadri SM, Chowdhary QA (2003) Metastasis of solid tumors in bone marrow: a study from Kashmir India. J Clin Pathol 56:803-804

11. Finklestein JZ, Ekert H, Issacs H, Higgins G (1970) Bone marrow metastasis in children with solid tumors. Am J Dis Child 119(1):49-52

12. Penchasky L (1984) Bone marrow biopsy in the metastatic work up of solid tumors in children. Cancer 54:1447-1448. doi: 10.1002/1097-0142(19841001)54:7<1447:AID-CNCR28205407 $37>3.0 . \mathrm{CO} ; 2-8$

13. Ingle JN, Tormey DC, Bull JM, Simon RM (1977) Bone marrow involvement in breast cancer: Effect on response and tolerance to combination chemotherapy. Cancer 39:104-111 